\title{
以基本科学素养为核心的无机及分析化学实验混合式教学实践
}

\author{
单丽伟，余瑞金，蒲亮，王文己，郁尧，龚宁 ${ }^{*}$ \\ 西北农林科技大学化学与药学院, 陕西杨凌 712100
}

\begin{abstract}
摘要: 贯彻 “以学生为中心、提升基本科学素养” 的教学理念, 对实验课程内容及授课形式进行重新设计; 建设了实验 教学课件、操作视频、虚拟仿真实验、试题库、拓展阅读素材等教学资源; 并通过超星学习通在 92 个班级开展线上线下 混合式教学实践。本课程改革极大地激发了学生的积极性与主动性, 潜移默化中提升了学生的科学素养, 取得了良好的 教学效果。为相关实验课程的教学改革提供了有益参考。
\end{abstract}

关键词: 无机及分析化学实验; 基本科学素养; 混合式教学; 课程资源建设

中图分类号: G64; O6

\section{The Blended Teaching Practice of "Inorganic and Analytical Chemistry Laboratory" with the Core of Basic Scientific Literacy}

\author{
Liwei Shan, Ruijin Yu, Liang Pu, Wenji Wang, Yao Yu, Ning Gong * \\ College of Chemistry \& Pharmacy, Northwest A \& F University, Yangling 712100, Shaanxi Province, China.
}

\begin{abstract}
Following the philosophy of "student-centered and improving basic scientific literacy", the contents and teaching forms of the laboratory course were redesigned, and the teaching resources such as the laboratory courseware, the experimental procedure video, the virtual simulation experiment, the test question bank and expanded reading materials were constructed. Blended online/offline teaching practice was carried out for 92 classes through the Super Star Learning platform. The reform has greatly stimulated the enthusiasm and initiative of students, imperceptibly improved students' scientific literacy and achieved good teaching effects. It also provides a useful reference for the teaching reform of related laboratory courses.
\end{abstract}

Key Words: Inorganic and analytical chemistry laboratory; Basic scientific literacy; Blended teaching; Curriculum resources construction

什么是科学素养? 科学素养是个体或群体在学习和应用科学知识过程中养成的内在品质, 是自 觉运用科学知识、科学方法认识、解释和解决实际问题的内在驱动力。美国学者米勒将科学素养的 内涵归结为三个方面 ${ }^{[1,2]}$ : (1) 对以科学概念和科学术语为核心的科学知识的了解与理解。(2) 对科 学研究过程和科学方法的理解和掌握。(3) 认识并了解科学技术对个人和社会生活的影响。科技创 新是驱动人类社会进步的核心动力, 提升全民科学素养是创新型社会发展的必然要求。作为未来社 会的主人公和建设者, 提升大学生科学素养已成为当代大学生教育的核心目标。

无机及分析化学实验是农林类高校多数专业开设的必修基础课, 也是大学生接触的第一门化学 类实验课。该课程不仅承担着加深理论知识理解、增强专业实验理论和基本实验技能学习的职责,

收稿: 2021-02-26; 录用: 2021-06-18; 网络发表: 2021-07-13

“通讯作者, Email: gongningcn@163.com

基金资助: 西北农林科技大学 2019 年教学改革研究项目(JY1903166); 西北农林科技大学 2020 年一流本科课程建设项目; 西北农林科技大学化 学与药学院 2020 年课程思政教学改革研究项目 
同时也肩负着培养大学生基本科学素养的职责。因而, 以基本科学素质养成为核心目标, 改革无机 及分析化学实验课程教学内容和教学模式、提高课程教学质量, 对农林类高校大学生培养质量的提 升具有重要意义。

\section{1 无机及分析化学实验教学中存在的问题及对策}

教学过程未能突出 “以学生为中心, 提升基本科学素养” 的教学理念。在传统实验教学过程中, 学生经常处于被动角色, 按部就班、甚至应付式完成实验课。古希腊哲学家苏格拉底说 “教育不是 灌输知识, 而是点燃火焰”, 因而以 “学生为中心” 激发学生的学习兴趣, 提高学生的自主学习能 力已成为当前教育模式改革的重中之重 ${ }^{[3]}$ 。如前所述, 提升科学素养已成为当代大学生教育的核心 目标。事实上, 实验课堂是培养学生基本科学素养的理想场所, 特别是对科学研究过程和科学方法 的理解和掌握。如何观察记录实验现象、分析数据、推导归纳结论、撰写实验报告、开展团队协作 等均是科学素养提升的重要环节。在以往的实验教学中我们也强调实验技能的培养, 但实验技能只 是科学素养的一部分。要解决上述问题, 需要对课程内容进行重新审视和规划、通过教学资源的精 心制作、教学模式的合理运用、教学流程和课程评价的严格执行, 才能最终培养出具备基本科学素 养的专业人才。

学生学习基础和教师授课能力参差不齐。西北农林科技大学每年参加该课程学习的学生约 3000 人, 他们来自 12 个学院的 24 个专业, 具有不同学习背景, 对实验课的接触和了解程度有很大差异, 因而学习和掌握实验原理和实验技能的快慢有很大落差。同时, 该课程专职教师人数相对不足, 需 要聘请相关专业教师及研究生参与教学, 部分代课教师对教学内容理解不到位, 教学要求和成绩评 定标准不统一, 造成教学效果参差不齐。对于前者需要建设并充分利用网络教学资源, 以便学生根 据自己的能力和节奏进行学习。对于后者则迫切需要建立一套标准化的实验教学体系, 实现教学内 容、教学模式和课程评价体系的统一。

\section{2 课程内容的改革与设计}

为了融汇 “以学生为中心, 提升基本科学素养” 的教学理念, 适应线上线下混合式教学的特 点 ${ }^{[4,5]}$, 我们对无机及分析化学实验教学内容进行了重新设计。本实验课程计划48学时, 其中线上教 学内容 8 学时, 包括实验安全教育、实验数据处理、原子分子结构虚拟仿真三部分。线上线下混合教 学内容 40 学时, 包括物质性质、滴定分析、仪器分析、分离纯化等实验, 具体安排见表 1 。通过上述 实验, 不仅培养学生基本实验操作技能和实验数据处理与报告撰写能力, 同时潜移默化地提高学生 的科学素养。不同类型的实验对科学素养的养成具有一定的针对性, 如物质性质实验注重培养学生 观察记录实验的能力; 定量分析实验注重培养严谨求实、精益求精的实验作风; 实事求是、团队合 作、安全意识和环保意识的培养则贯穿在实验教学的各个环节。

\section{3 课程资源建设}

以重新规划后的课程内容为基础进行网络教学资源建设。制作无机及分析化学实验教学课件 16 个(图1); 并录制对应的授课视频, 视频时长为5-20 min, 便于学生课前自主在线学习。录制实验基 本操作和常规实验仪器使用视频 25 个, 包括常规仪器(滴定管、移液管、容量瓶、分析天平、分光光 度计、酸度计等)使用及基本实验操作(溶液配制、离心操作、滴定操作、称量操作、半滴停靠、常压 过滤、减压过滤等); 构建了以选择题、判断题、简答题为主要题型的试题库(图1), 一共包括1072道 测试题, 覆盖了实验室安全知识、实验数据处理、仪器使用、基本操作及每个实验的具体内容。根 据以往的教学经验, 针对每一个实验设计讨论题、思考题及实验案例, 利用讨论题目开展课堂小组 讨论, 撰写报告时完成思考题和实验案例, 对实验进行总结和反思。将上述各种素材整合放入超星 学习通教学平台 ${ }^{[6,7]}$, 以便教师展开线上线下混合教学。 
大学 化学 Univ. Chem. 2021, 36 (12), 2102053 (3 of 5)

表1 无机及分析化学实验课程内容设计

\begin{tabular}{|c|c|c|c|c|}
\hline 实验内容 & 实验类型 & 学时 & 授课模式 & 基本科学素养 ${ }^{*}$ \\
\hline 1. 课程介绍及化学实验室安全知识 & 安全教育 & 3 & 线上 & \\
\hline 2. 有效数字及数据处理 & 数据处理 & 2 & 线上 & 实事求是的科学精神 \\
\hline 3. 原子及分子结构虚拟仿真实验 & 虚拟仿真 & 3 & 线上 & 精益求精的实验态度 \\
\hline 4. 性质综合实验一(酸碱反应、沉淀溶解平衡、胶体溶液) & 性质实验 & 5 & 线上线下 & 精诚合作的团队精神 \\
\hline 5. 性质综合实验二(氧化还原反应、配合物性质、化学反应 & 性质实验 & 5 & 线上线下 & 贯穿始终的实验安全意识 \\
\hline 速率与化学平衡) & & & & 贯穿始终的环境保护意识 \\
\hline 7. 酸碱标准溶液配制及食醋中醋酸含量的测定(酸碱滴定废 & 定量分析 & 5 & 线上线下 & \\
\hline 水处理) & & & & 实验现象的观察与记录 \\
\hline 8. 混合碱的组成与含量测定 & 定量分析 & 5 & 线上线下 & \\
\hline 9. 有关化学常数的测定(阿伏伽德罗常数、醋酸解离常数) & 常数测定 & 5 & 线上线下 & 化学实验基本操作技能的培养 \\
\hline 10. 自来水硬度的测定 & 定量分析 & 5 & 线上线下 & 常规仪器使用方法的掌握 \\
\hline 11. 邻二氮菲分光光度法测定铁的含量 & 仪器分析 & 5 & 线上线下 & 头验报哇的撰与及反思 \\
\hline 12. 粗食盐的提纯 & 分离提纯 & 5 & 线上线下 & \\
\hline
\end{tabular}

*所列出的科学素养与左侧的实验内容不是一一对应。一个实验可同时培养多种科学素养, 同一种科学素养需要通过多个实验反复强化。

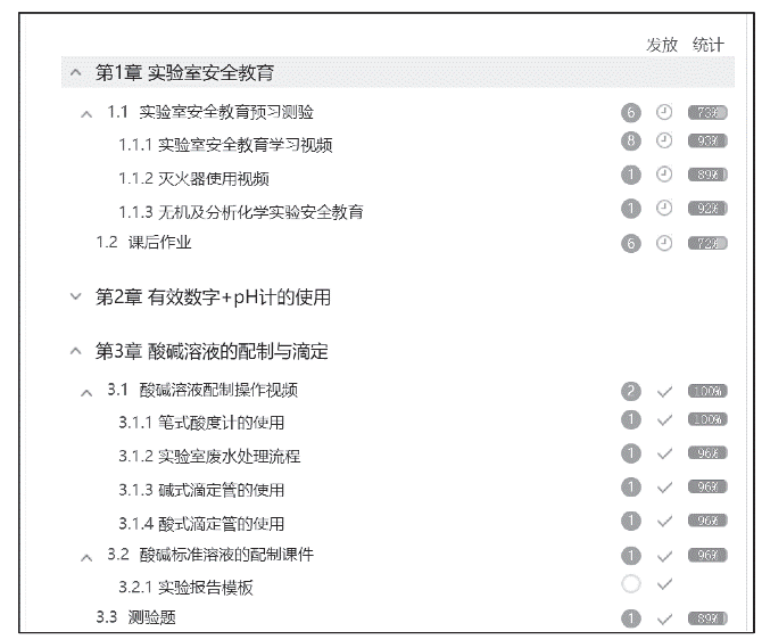

(A)

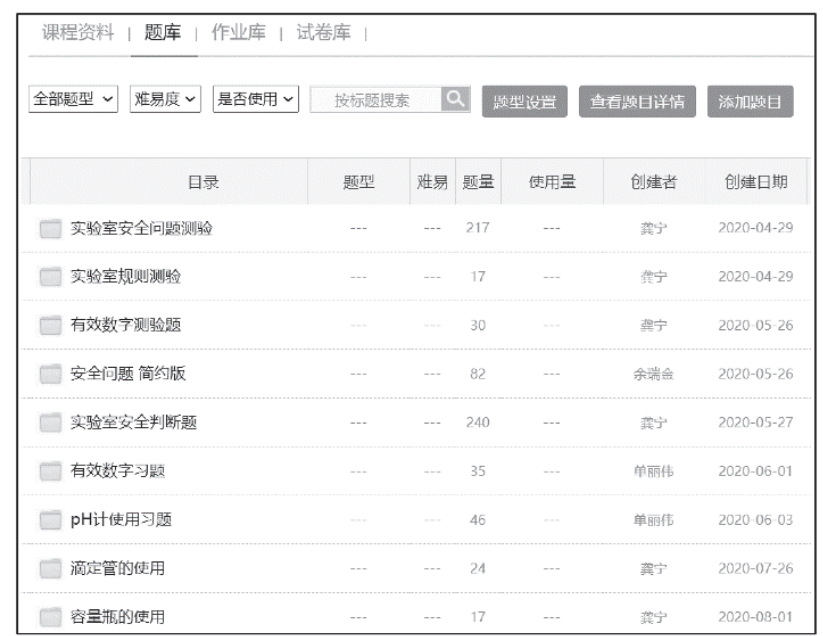

(B)

图1 超星学习通网络教学平台建设

(A) 课程任务列表; (B) 题库列表

\section{4 线上线下混合教学实践}

理论课可采用线上教学(如中国大学慕课)、线上线下混合教学或线下教学, 实验课则更适合线 上线下混合式教学或线下教学 ${ }^{[8-10]}$ 。2018-2019年, 我们在部分班级中开展无机及分析化学实验课程 的线上线下混合式教学, 2020年则利用超星学习通平台将该教学模式推广到全校92个教学班。授课 前首先建立班级 $\mathrm{QQ}$ 群, 以便教师和学生沟通, 发布课程公告, 开展课程直播; 学生利用学习通进行 在线学习、观看操作视频、开展线上讨论、完成在线练习和测试、提交实验报告等。本实验课程共 安排了11次课, 其中课程介绍及安全知识教育、数据处理、虚拟仿真三次课通过线上教学完成, 采 用学生自主学习或网络直播方式开展; 其他8次实验课均采用线上线下混合式教学。 
线上线下混合式教学的实施包括三个环节: 课前预习、课堂学习和课后提升。课前预习环节中, 需要学生自主阅读实验指导书、观看相关实验讲授视频和操作视频、完成在线预习测试并撰写预习 报告。课堂学习环节中, 教师首先检查学生的预习效果, 然后讲解实验重点难点及实验操作注意事 项, 演示关键的操作流程和仪器使用方法; 如若对实验原理或操作细节存在疑问, 学生可通过学习 通平台或扫描教材上的二维码观看相关视频; 然后学生进行自主实验, 认真观察实验现象并如实记 录实验数据, 完成规定实验内容; 教师则及时纠正学生的错误操作, 同时协助学生找出错误原因并 记录学生的实验表现; 实验完成后, 学生须经教师检查后将实验原始数据、图片及视频资料上传网 络教学平台; 师生共同讨论实验中出现的问题, 并总结经验教训。实验课后, 学生须及时分析处理 数据, 得出实验结果及结论, 总结实验心得和收获, 按时完成并在线提交实验报告, 完成学习效果 评价在线测试; 教师则须完成实验报告评价, 及时反馈督促学生纠错。课程内容的改革和线上线下 混合式教学模式的运用极大地激发了学生的自主学习热情, 在线视频和在线测试的参与度达到了 $92 \%$ 以上(图2)。由于课前的充分预习及实验操作与仪器使用视频观看的便捷性, 学生对实验原理理 解的透彻性和实验操作技能的规范性均优于以往。

建立统一的实验课程考核标准是实现教学目标的重要一环。实验课程成绩的评价不同于理论教 学, 无法仅通过考试完成, 更应该注重过程性评价。如何实现过程性评价一直是个难题, 线上线下 混合式教学和各种新兴教育技术的应用使过程性评价的量化成为可能。我们初步建立了该实验课成 绩考核标准, 该标准包括在线学习 $(15 \%)$ 、在线测试(15\%)、实验操作 $(40 \%)$ 和实验报告 $(30 \%)$ 四部分 内容(图3)。前两部分可通过网络教学资源的使用度和在线测试成绩综合给出。实验操作水平和实验 报告评价则以相应的评分细则由任课教师给出, 实现课程评价体系的标准化和客观性。

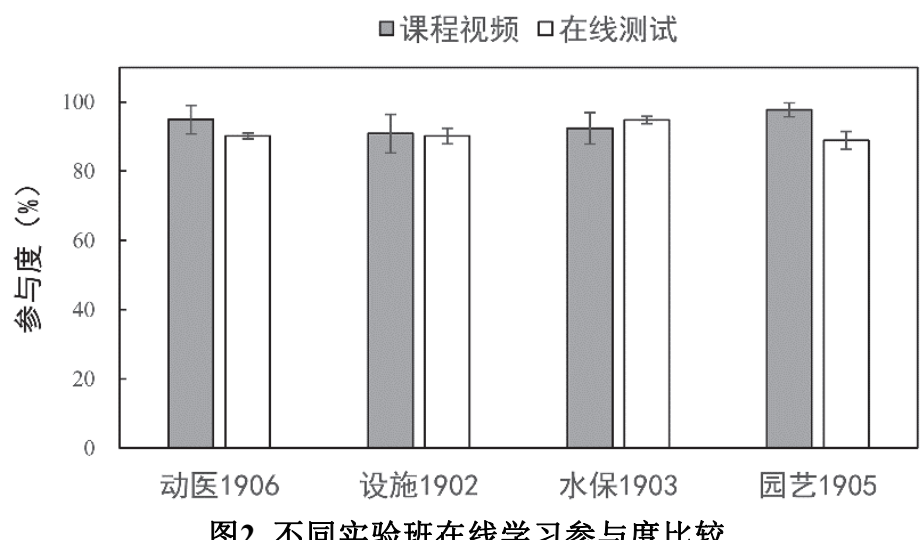

图 2 不同实验班在线学习参与度比较

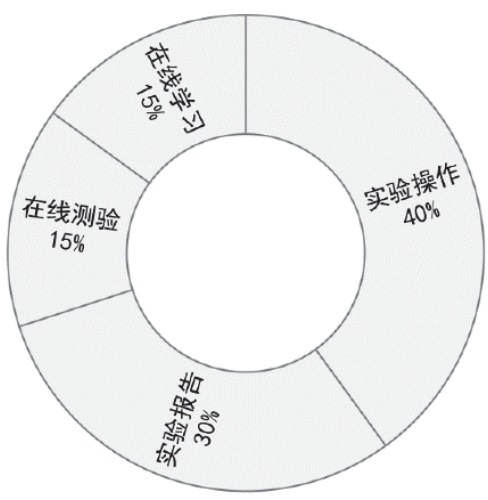

图3 实验成绩考核标准

\section{5 科学素养在实验课程中的融会贯通}

在课程改革实践中，我们将 “提升科学素养”这一核心目标融汇在实验课程内容规划、新形态 教材建设 ${ }^{[11]}$ 、课程资源建设、线上线下混合教学的各个环节。如前所述, 科学素养的内涵包括三个 方面, 其中以科学概念和科学术语为核心的科学知识的掌握主要通过理论教学完成; 科学研究过程 和科学方法的理解和掌握则主要通过实验课和实践来实现; 科技对个人和社会的影响则需要在理论 课、实验课和实践中不断强化。也有学者根据上述内涵, 将科学素养的组成归结为三个同心圆 ${ }^{[12]}$, 其中最核心的是科学精神、态度和科学观, 中间部分是科学知识、技能、方法和能力, 最外围是科 学行为和习惯。

数据的采集与处理是科学素养培养的重要环节。下面以定量分析实验中有效数字及数据处理为 例, 说明如何引导学生具备 “量” 的意识, 将有效数字取舍及数据处理训练贯穿定量分析实验始终。 实验课前要求学生在线学习有效数字及数据处理相关内容, 完成相关知识测试, 初步掌握有效数字 
的定义、取舍原则及运算规则, 以及数据的正确读取、记录及分析处理方法。在实验课堂教学中, 任课教师通过自己或学生示范, 强调数据采集及记录方法, 如样品称量过程中的数据记录、滴定分 析中滴定管的读法、分光光度计和 $\mathrm{pH}$ 计的数值读取等; 同时学生也可通过扫描实验教材上的二维码 反复查看仪器使用方法和数据采集技巧。实验操作过程中, 教师会随时纠错并对学生的各种疑问进 行解答。实验结束, 学生须将自己的实验数据记录本交给教师进行检查, 教师检查后在线提交数据; 如果实验数据不可用, 则要求学生重新补做实验。课后学生将实验报告提交至学习通平台, 教师将 数据记录处理结果作为实验报告考核的主要指标, 并及时将问题反馈给学生并要求学生进行修改补 充。通过反复的练习和强化, 使学生的 “量化” 意识和技能成为一种自觉习惯。

在实验教学环节中, 我们首先要求学生以事实为依据, 不断强化实事求是、勇于创新、精益求 精的科学精神。其次, 通过实验课教学过程使学生了解科学研究的基本过程, 培养科学的行为方式 和思维模式, 掌握科学研究的基本方法和技能。同时, 科学技术是把双刃剑, 不合理、不恰当和恶 意的使用会给人类带来灾难。作为科学素养的一部分, 我们在实验课程中不断强化学生的安全意识 和环保意识。要求学生实验后对产生废液的组分进行分析, 并对实验废水进行初步处理和处置, 让 安全和环保意识渗透到教学的各个环节, 不断增强学生的社会责任感。

\section{6 结语}

大学教育的目标不仅是知识和技能的传授, 更重要的是良好专业和科学素养的塑造。无机及分 析化学实验课是高等院校理工农医类专业开设的必修基础课, 也是大学生接触的第一门化学类实验 课, 是从头培养学生基本专业和科学素养的重要课堂。我们通过数年的探索实践, 将线上线下混合 式教学应用于无机及分析化学实验课, 将科学素养的提升贯穿在实验课教学的各个环节, 实现了教 材、教学资源的叠加式改进, 激发了学生学习的主动性和热情, 显著提升了学生科学素养和实验教 学效果。

\section{参 考 文 献}

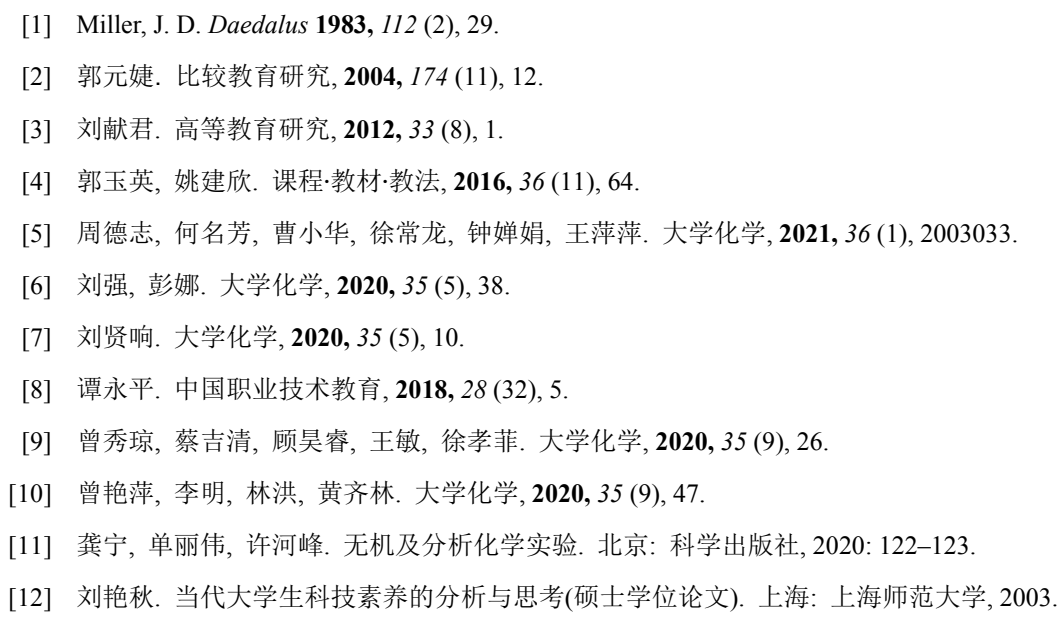

